\title{
Design and Implementation of a Haptic Device for Training in Urological Operations
}

\author{
Kostas Vlachos, Evangelos Papadopoulos, Senior Member, IEEE, and Dionissios N. Mitropoulos
}

\begin{abstract}
Virtual reality is becoming very important for training medical surgeons in various operations. Interfacing users with a virtual training environment requires the existence of a properly designed haptic device. This paper presents the design and implementation of a new force feedback haptic mechanism with five active degrees of freedom (DOFs), which is used as part of a training simulator for urological operations. The mechanism consists of a 2-DOF, 5-bar linkage, and a 3-DOF spherical joint, designed to present low friction, inertia and mass, and to be statically balanced. The device is suitable for the accurate application of small forces and moments. All five actuators of the haptic device are base-mounted dc motors and use a force transmission system based on capstan drives, pulleys, and tendons. The paper describes the overall design and sizing considerations, the resulting kinematics and dynamics, the force feedback control algorithm, and the hardware employed. Experimental results are provided.
\end{abstract}

Index Terms-Force feedback, haptic devices, training medical simulators, urological operations.

\section{INTRODUCTION}

D URING the past several years, research on the use of virtual reality in medicine has rapidly increased. Because of the recent developments in software and hardware, it is now possible to create realistic simulation environments for educational purposes in medicine [1]. Training in a simulated environment has significant advantages over the traditional medical training. It is less expensive and results in efficient training and practice in complicated procedures [2]. Training on patients can result in serious damages and lawsuits while training on animals becomes an undesirable alternative for ethical and economical reasons. Also, the existence of a training simulator increases the availability of the training environment, allows an easier evaluation of the performance of the trainee, and can be used to introduce various operation scenarios or situations.

Development of a useful and sufficiently realistic surgical simulator requires the use of two core technologies, such as graphical simulation in a virtual reality context and haptic tech-

Manuscript received June 15, 2002; revised January 16, 2003. This paper was recommended for publication by Editor R. Taylor upon evaluation of the reviewers' comments. This work was supported by the PENED and PRAXE programs of the Hellenic General Secretariat for Research and Technology. This paper was presented in part at the IEEE International Conference on Robotics and Automation, Washington, DC, April, 2002.

K. Vlachos and E. Papadopoulos are with the Department of Mechanical Engineering, National Technical University of Athens, 15780 Athens, Greece (E-mail: kostaswl@central.ntua.gr; egpapado@central.ntua.gr).

D. N. Mitropoulos is with the Department of Urology, Faculty of Medicine, National \& Kapodistrian University of Athens, 11527 Athens, Greece (e-mail: dmp@otenet.gr).

Digital Object Identifier 10.1109/TRA.2003.817064 nologies, including force feedback. While a realistic visual representation of human anatomy and tissue deformation is very important, the ability to command the graphic environment and interact with it through the feel of the forces and torques is also of paramount importance. In order to implement such a feel, haptic devices are used.

The earliest haptic devices were of the master-slave type, and were used for telemanipulation of hazardous materials [3]. In the last 15 years, the availability of powerful computers have resulted in the proliferation of virtual reality systems. In these systems, the slave is replaced by a computational model and its motion appears graphically.

Today, one can distinguish two trends in the development of medical simulators. The first is described by the use of general-purpose haptic devices, like the Phantom or the Freedom-7 [4]-[6]. The Phantom is a five-bar parallel mechanism with three or six degrees of freedom (DOFs) which is designed as a general-use haptic interface [7]. The Phantom 1.5/6-DOF and the Phantom Premium 6-DOF prototype have six active DOFs, but are designed mainly for virtual prototyping, virtual assembly, etc. The actuators are mounted on the moving parts of the mechanism, the mechanism inertia is relative large, and the acting forces and workspace are not suitable for the operation described in this paper [8]. A training simulator for the diagnosis of prostate cancer using the Phantom haptic interface is described in [9]. The same interface is used in the Spine Biopsy Simulator described in [10], and as part of a robotic haptic system that provides assistance to people with disabilities [11]. Freedom-7 is a haptic device with six DOFs for translation and rotation, plus a seventh for the movement of tools like scissors and forceps. It has six active DOFs and is also designed for general haptic applications [12].

The second trend is described by the use of haptic devices designed for a specific operation. The PantoScope belongs to this category. It is a 4-DOF mechanism for force reflection in a laparoscopic surgery simulation environment [13]. The Laparoscopic Impulse Engine is another haptic device for laparoscopic and endoscopic procedures with five DOFs, of which three provide force feedback. VIRGY is an endoscopic surgery simulator, which uses the PantoScope haptic device [14]. The "Karlsruhe Endoscopic Surgery Trainer" and the "Virtual Endoscopic Surgery Training" (VEST) are training simulators specialized in laparoscopic operations using the software system KISMET [15]. Three types of force-reflecting interfaces can be connected to KISMET, the Laparoscopic Impulse Engine, the Phantom, and the HIT Force Feedback Device designed in Forschungszentrum Karlsruhe. All these force feedback devices have three active DOFs only. 


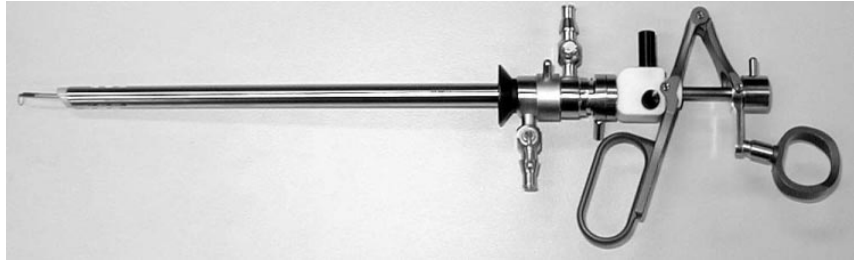

Fig. 1. Endoscope for urological operations.

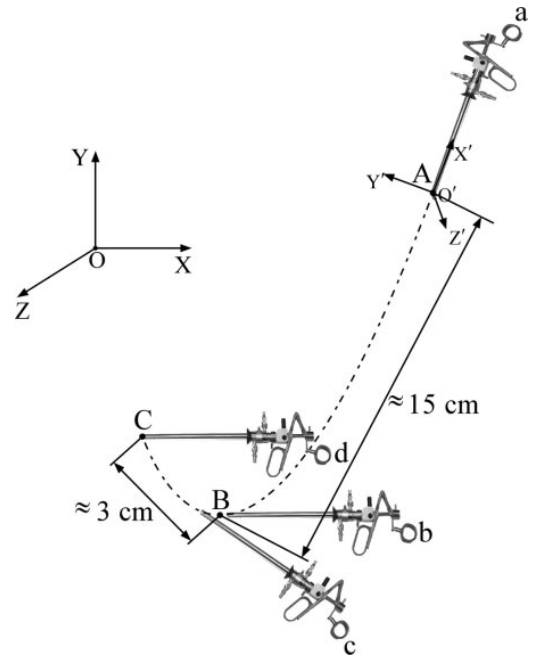

Fig. 2. Endoscope endpoint path and orientation during an operation.

This paper presents a new 5-DOF haptic device for training in male urological operations, of which all five DOFs are active. The mechanism allows 3-DOF tool orientation motions and 2-DOF translation motions. The orientation pitch-roll-yaw DOFs are decoupled from themselves and from the mechanism translational DOF. Unlike other haptic devices, in which the maximum forces or torques are of prime importance, here it is very important to have a device that can reproduce faithfully very small forces and torques, like those that appear in urological operations. Therefore, a major effort was placed in designing the mechanism such that it is characterized by small friction and inertia. The paper analyzes the kinematics and the dynamics of the mechanism, describes its mechanical design, and presents experimental results on its performance. Finally, it describes a control law and implementation solutions.

\section{REQUIREMENTS}

During a urological operation on a male, the surgeon has to insert a long cylindrical endoscope, see Fig. 1, until its endpoint reaches the patient's bladder. A minicamera at the endoscope endpoint provides optical information about the endpoint location and the condition of the tissues.

Observations of actual operations on humans and animals showed that a urological operation is divided into two phases, i.e., the insertion phase and the main operation.

During insertion, the endoscope follows a path such as the typical one shown in Fig. 2. The surgeon moves the tip of the endoscope from the insertion point $\mathrm{A}$ to the final point $\mathrm{C}$, via
TABLE I

HAPTIC DEVICE WORKSPACE REQUIREMENTS

\begin{tabular}{lr}
\hline \hline Translation along the $X$ axis & $10 \mathrm{~cm}$ \\
Translation along the $Y$ axis & $10 \mathrm{~cm}$ \\
Translation along the $Z$ axis & $0 \mathrm{~cm}$ \\
Rotation about the $X^{\prime}$ axis & $\pm 180^{\circ}$ \\
Rotation about the $Y^{\prime}$ axis & $\pm 30^{\circ}$ \\
Rotation about the $Z^{\prime}$ axis & $\pm 30^{\circ}$ \\
\hline \hline
\end{tabular}

TABLE II

FORCE/TORQUE UPPER LIMITS DURING AN OPERATION

\begin{tabular}{lr}
\hline \hline Force along the $X$ axis & $4.50 \mathrm{~N}$ \\
Force along the $Y$ axis & $4.50 \mathrm{~N}$ \\
Torque about the $X^{\prime}$ axis & $10.00 \mathrm{mNm}$ \\
Torque about the $Y^{\prime}$ axis & $150.00 \mathrm{mNm}$ \\
Torque about the $Z^{\prime}$ axis & $150.00 \mathrm{mNm}$ \\
\hline \hline
\end{tabular}

an intermediate point B, see Fig. 2. At point B, the endoscope orientation changes without translation, so as to align the entire urethra and continue the insertion phase without traumas. The corresponding endoscope configurations labeled by a, b, c, d, are shown in Fig. 2.

When the endpoint of the endoscope reaches the bladder (point $\mathrm{C}$ in Fig. 2), the surgeon inserts through the endoscope a mechanism with a scissor-like handle and begins the second phase. This phase is the main operation in which tissue removal occurs. During this phase, the movements of the endoscope are mainly rotational.

Observations of actual operations showed that although the endoscope can have any orientation in a cone, its endpoint translations occur on a plane of symmetry of the patient. Therefore, a 5-DOF haptic mechanism is needed, with two translational and three rotational DOFs.

The actual kinematic requirements that define the minimum workspace of the haptic interface are shown in Table I. These were found by actual observations of sample urological operations. The workspace is defined with respect to a reference coordinate frame $(O, X, Y, Z)$, where $O$ is the base point of the mechanism and $X-Y$ the plane of symmetry of the patient. The rotations are defined with respect to an endoscope body-fixed coordinate frame $\left(O^{\prime}, X^{\prime}, Y^{\prime}, Z^{\prime}\right)$. The length along the endoscope path $\mathrm{ABC}$ in Fig. 2 is about $20 \mathrm{~cm}$.

The forces that the surgeon feels during the operation are small but of great importance, because they provide the necessary feedback for the successful accomplishment of the operation. In order to reproduce these small forces, the haptic mechanism must be balanced, have low inertia, low friction, no backlash, and be absolutely backdriveable.

The upper limits of forces and torques during a urological operation were measured in collaboration with specialist surgeons and are shown in Table II. However, the minimum forces and torques actually felt during an operation can be a fraction of these upper limits. This makes the design of a haptic device for such operations quite challenging. 


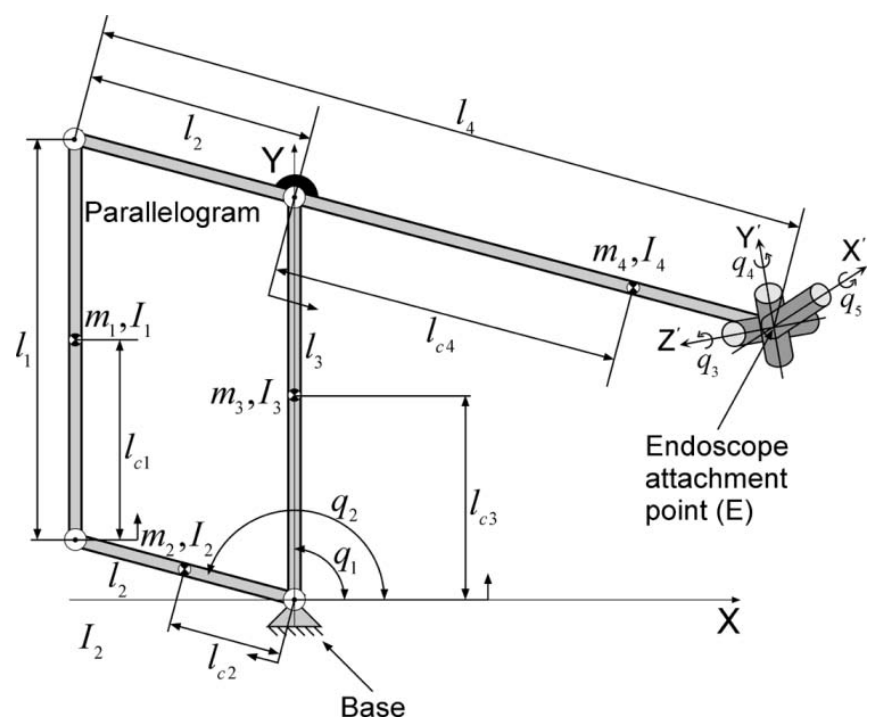

Fig. 3. Schematic view of a 5-DOF haptic mechanism and its kinematic and dynamic parameters.

\section{KINEMATICS \& DYNAMICS}

To develop the device, first a basic mechanism configuration that could satisfy, in principle, the identified requirements had to be selected. To this end, a hybrid design that consists of a closed five-bar parallel linkage providing two translational DOFs in series to a spherical joint with three rotational DOFs was chosen, see Fig. 3. The mechanism origin is located at the joint linking links 2 and 3 and not at the joint linking links 1 and 2. For the typical path shown in Fig. 2, this choice results in better static and dynamic characteristics. The endoscope is attached at point $\mathrm{E}$ in Fig. 3, i.e., the point at which the three axes of the last three joints intersect. For design reasons, both the differential kinematics and the dynamics of the mechanism are important and developed next.

The differential kinematics for this mechanism relate the endpoint Cartesian velocities and angular velocities contained in the vector $\mathbf{v}=\left[\dot{x}, \dot{y}, \omega_{x}, \omega_{y}, \omega_{z}\right]^{T}$ to the joint rates $\dot{\mathbf{q}}=\left[\dot{q}_{1}, \dot{q}_{2}, \dot{q}_{3}, \dot{q}_{4}, \dot{q}_{5}\right]^{T}$

$$
\begin{aligned}
{\left[\begin{array}{c}
\dot{x} \\
\dot{y} \\
\omega_{x} \\
\omega_{y} \\
\omega_{z}
\end{array}\right]=} & {\left[\begin{array}{ccccc}
-l_{3} s_{1} & l_{4} s_{2} & 0 & 0 & 0 \\
l_{3} c_{1} & -l_{4} c_{2} & 0 & 0 & 0 \\
0 & 0 & 0 & s_{2+3} & -c_{2+3} c_{4} \\
0 & 0 & 0 & -c_{2+3} & -s_{2+3} c_{4} \\
0 & 1 & 1 & 0 & -s_{4}
\end{array}\right] } \\
& \times\left[\begin{array}{c}
\dot{q}_{1} \\
\dot{q}_{2} \\
\dot{q}_{3} \\
\dot{q}_{4} \\
\dot{q}_{5}
\end{array}\right] \\
\mathbf{v}= & \mathbf{J}_{1}(\mathbf{q}) \dot{\mathbf{q}}
\end{aligned}
$$

where $s_{i}$ is the $\sin ($.$) of angle q_{i}, c_{i}$ is the $\cos ($.$) of angle q_{i}$, $s_{i+j}$ is the $\sin ($.$) of angle q_{i}+q_{j}$, etc.

The translational five-bar mechanism becomes singular when $q_{1}=q_{2}$ or $q_{1}=q_{2} \pm \pi$. The spherical joint, which is of the $Z$ - $Y$ - $X$ type, becomes singular when $q_{4}= \pm \pi / 2$. However, these configurations are not possible for the actual mechanism, due to endoscope-induced mechanical constraints. Therefore, the actual device is free of singularities in its workspace.

The joint rates are related to actuator rates $\dot{\theta}$ via matrix $\mathbf{J}_{2}$

$$
\dot{\mathbf{q}}=\mathbf{J}_{2}\left[\dot{\theta}_{1}, \dot{\theta}_{2}, \dot{\theta}_{3}, \dot{\theta}_{4}, \dot{\theta}_{5}\right]^{T}=\mathbf{J}_{2} \dot{\theta}
$$

This matrix depends on the transmission technique used. Then, the endpoint velocities are written as

$$
\mathbf{v}=\mathbf{J}_{1}(\mathbf{q}) \mathbf{J}_{2} \dot{\theta}=\mathbf{J}(\mathbf{q}) \dot{\theta} .
$$

Employing a Lagrangian formulation yields the following mechanism equations of motion:

$$
\mathbf{M}(\mathbf{q}) \ddot{\mathbf{q}}+\mathbf{V}(\mathbf{q}, \dot{\mathbf{q}})+\mathbf{G}(\mathbf{q})=\boldsymbol{\tau}^{\prime}+\mathbf{J}_{1}^{T} \mathbf{F}_{T}
$$

In (4), $\mathbf{M}(\mathbf{q})$ is the mechanism $5 \times 5$ mass matrix, $\ddot{q}$ is the joint accelerations vector, $\mathbf{V}(\mathbf{q}, \dot{\mathbf{q}})$ is the vector of nonlinear velocity terms, and $\mathbf{G}(\mathbf{q})$ is the gravity vector. The vector $\boldsymbol{\tau}^{\prime}$ contains joint input torques, while the vector $\mathbf{J}_{1}^{T} \mathbf{F}_{T}$ resolves the forces and torques applied by the endoscope to the mechanism, to its five joints.

For design and control reasons, it is useful to eliminate the gravity terms by statically balancing the mechanism. If these terms were present, then the controller would have to provide control torques to balance the mechanism, since these terms do not exist in an actual operation. If the controller had to compensate for the gravity terms, then an accurate identification of these terms would be needed, while the actuator size and, therefore, rotor inertia would have been larger.

Assuming that the spherical joint center of mass coincides with the intersection of the last three axes, the nonzero gravity terms for the unbalanced mechanism are

$$
\begin{gathered}
g_{1}(\mathbf{q})=g c_{1}\left(m_{1} l_{c^{1}}+m_{3} l_{c^{3}}\right. \\
\left.+\left(m_{4}+m_{5}+m_{6}+m_{7}\right) l_{3}\right) \\
g_{2}(\mathbf{q})=g c_{2}\left(m_{1} l_{2}+m_{2} l_{c^{2}}-m_{4} l_{c^{4}}\right. \\
\left.-\left(m_{5}+m_{6}+m_{7}\right) l_{4-2}\right)
\end{gathered}
$$

where $l_{4-2}=l_{4}-l_{2}$. These terms can be eliminated if two balancing weights are used with masses $m_{b 2}$ and $m_{b 3}$ equal to

$$
\begin{aligned}
m_{b^{3}} & =\frac{\left(m_{3} l_{c^{3}}+\sum_{i=4}^{7} m_{i} l_{3}-m_{c^{3}} l_{c b^{3}}+m_{1} l_{c^{1}}\right)}{l_{b^{3}}} \\
m_{b^{2}}= & \frac{\left(m_{4} l_{c^{4}}+\sum_{i=5}^{7} m_{i} l_{4-2}-m_{1} l_{2}-m_{c^{2}} l_{c b^{2}}-m_{2} l_{c^{2}}\right)}{l_{b^{2}}} .
\end{aligned}
$$

The balancing weights are located on the extensions of links 2 and 3 , at distances $l_{b^{2}}$ and $l_{b^{3}}$ from the base point of the mechanism, respectively. These lengths were chosen so that mechanism balancing can be achieved without collisions while the total link inertia is minimized.

Using (1b) and its derivative

$$
\ddot{\mathbf{q}}=\mathbf{J}_{1}^{-1}\left(\dot{\mathbf{v}}-\dot{\mathbf{J}}_{1} \mathbf{J}_{1}^{-1} \mathbf{v}\right)
$$


and assuming a balanced mechanism, the equations of motion, as seen from the endoscope side and written for its tip motion, reduce to

$$
\tilde{\mathbf{M}} \dot{\mathbf{v}}+\tilde{\mathbf{V}}=\mathbf{J}^{-T} \boldsymbol{\tau}+\mathbf{F}_{T}
$$

with

$$
\tilde{\mathbf{M}}=\mathbf{J}_{1}^{-T} \mathbf{M} \mathbf{J}_{1}^{-1}, \quad \tilde{\mathbf{V}}=\mathbf{J}_{1}^{-T} \mathbf{V}-\mathbf{J}_{1}^{-T} \mathbf{M} \mathbf{J}_{1}^{-1} \dot{\mathbf{J}}_{1} \mathbf{J}_{1}^{-1} \mathbf{v}
$$

where $\tau$ is the motor torque vector. Notice that the effective mass matrix $\tilde{\mathbf{M}}$ is not constant due to the appearance of the Jacobian and the dependence of $\mathbf{M}$ to joint variables. Although it is not possible to make $\tilde{\mathbf{M}}$ a constant matrix, its structure is simpler if the original $\mathbf{M}$ is a constant matrix. Therefore, $\mathbf{M}$ is approximately made such a matrix by proper design.

\section{FIVE-BAR LinKAGE SIZING}

In computing the actual five-bar linkage dimensions, various conflicting requirements had to be taken into account. For example, the mechanism must be large enough to follow typical endoscope paths, such as the one shown in Fig. 2. At the same time, it must exhibit a small mass and inertia.

Since the main phase of the operation is characterized by rotations and small motions about point $\mathrm{C}$, see Fig. 2, it is desired that at this point the condition number of the linkage is small. An optimum condition number is not necessarily beneficial to the mechanism, since this number may become much worse at other configurations, and because it results in a larger-than-needed mechanism, with a larger mass and inertia.

It can be shown that the mechanism condition number is optimum when $l_{4-2} \equiv l_{4}-l_{2}=l_{1} \equiv l_{3}$ and $q_{2}-q_{1}=90^{\circ}$, while it increases when $l_{4-2} \neq l_{1}$ and $q_{2}-q_{1}=90^{\circ}$. To achieve good condition numbers, the following inequalities that must hold at all configurations are employed:

$$
\begin{aligned}
l_{1} & \leq l_{4-2} \leq 1.25 l_{1} \\
30^{\circ} & \leq q_{2}-q_{1} \leq 150^{\circ} \\
60^{\circ} & \leq q_{2, C}-q_{1, C} \leq 120^{\circ}
\end{aligned}
$$

where the subscript in $q_{i, C}, i=1,2$ denotes the values of the angles at point $\mathrm{C}$. The requirements at $\mathrm{C}$ are more stringent than those along the path, for the reason discussed above.

Another requirement is the result of implementation constraints, i.e., to avoid transmission tendon misplacement, the angle $\Psi$ that is formed by link 4 and the endoscope, see Fig. 4 , has to be bounded according to

$$
-80^{\circ} \leq \Psi \leq 80^{\circ}
$$

These inequalities must be taken into account to yield the minimum length for $l_{4-2}$ and the best path starting point for a typical path, see Fig. 2. In this way, the mass and inertia of the mechanism can be kept small. This is described next.

Since $\Psi=\theta(s)-\theta_{2}$, and $\theta_{2}=q_{2}-\pi$, see Fig. 4, (12) yields

$$
-80^{\circ}+\theta(s)+\pi \leq q_{2} \leq \pi+\theta(s)+80^{\circ}
$$

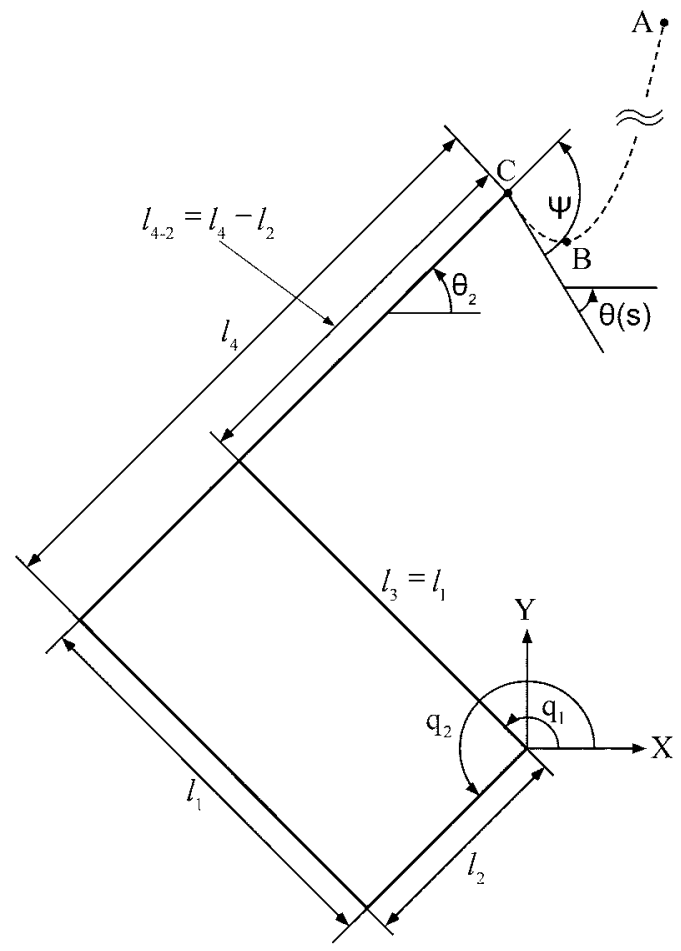

Fig. 4. Schematic view of the five-bar linkage and of a typical path to follow.

where $\theta(s)$ is the endoscope absolute angle with respect to the $X$ axis. A detailed path study reveals that this angle takes values between $-45^{\circ}$ and $70^{\circ}$. Equation (13) and these limits yield

$$
170^{\circ} \leq q_{2} \leq 215^{\circ}
$$

The five-bar linkage kinematics are described by

$$
\begin{aligned}
x_{E}+l_{4-2} \cos q_{2} & =l_{1} \cos q_{1} \\
y_{E}+l_{4-2} \sin q_{2} & =l_{1} \sin q_{1} .
\end{aligned}
$$

From (15), it follows that

$x_{E} \cos q_{2}+y_{E} \sin q_{2}=\frac{1}{2 l_{4-2}}\left(l_{1}^{2}-x_{E}^{2}-y_{E}^{2}-l_{4-2}^{2}\right)$.

Setting $y_{E} / x_{E}=\tan \omega$ and solving (16) for $q_{2}$ yields

$q_{2}=\omega-\arccos \left(\frac{\sin \omega}{2 l_{4-2} y_{E}}\left(l_{1}^{2}-x_{E}^{2}-y_{E}^{2}-l_{4-2}^{2}\right)\right)$.

Using (14) and (17), and after some manipulation, the following bounds are computed:

$$
\begin{aligned}
\omega-170^{\circ} & \geq \arccos \left(\frac{\sin \omega}{2 l_{4-2} y_{E}}\left(l_{1}^{2}-x_{E}^{2}-y_{E}^{2}-l_{4-2}^{2}\right)\right) \\
& \geq \omega-215^{\circ} .
\end{aligned}
$$

Equation (18) can be written as

$$
\begin{aligned}
& l_{4-2}^{2}+l_{4-2} \frac{2_{y_{E}} \cos \left(\omega-170^{\circ}\right)}{\sin \omega}+\left(x_{E}^{2}+y_{E}^{2}-l_{1}^{2}\right) \geq 0 \\
& l_{4-2}^{2}+l_{4-2} \frac{2_{y_{E}} \cos \left(\omega-215^{\circ}\right)}{\sin \omega}+\left(x_{E}^{2}+y_{E}^{2}-l_{1}^{2}\right) \leq 0 .
\end{aligned}
$$


TABLE III

MECHANISM LINK LENGTHS

\begin{tabular}{cc}
\hline \hline Link $1, l_{1}$ & $13.50 \mathrm{~cm}$ \\
Link $2, l_{2}$ & $7.50 \mathrm{~cm}$ \\
Link $3, l_{3}$ & $13.50 \mathrm{~cm}$ \\
Link $4, l_{4}$ & $23.00 \mathrm{~cm}$ \\
\hline \hline
\end{tabular}

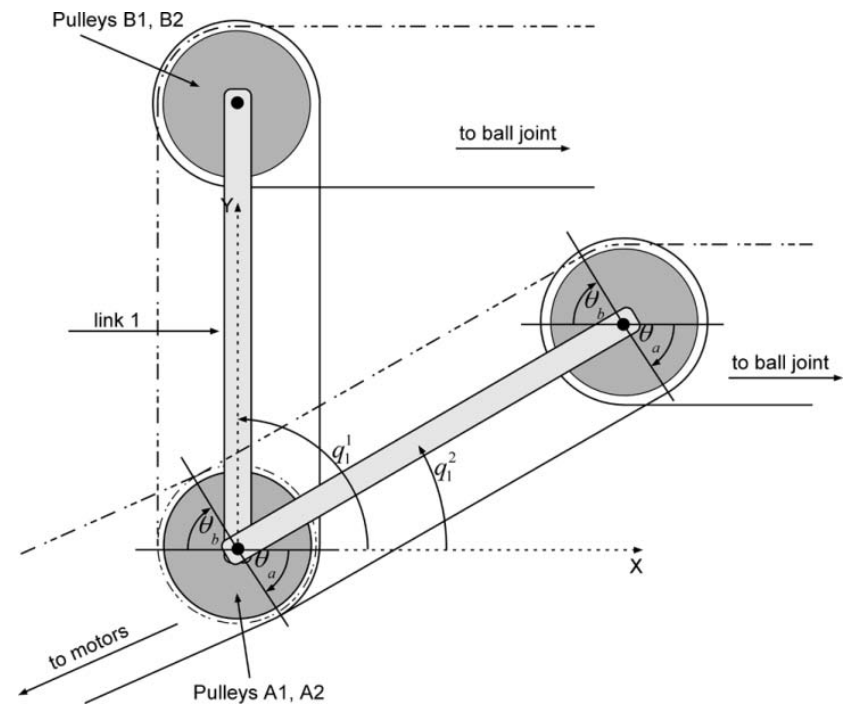

Fig. 5. Tendon transmission path design for constant tendon lengths.

The system of inequalities (9)-(11) and (19) is satisfied for a range of path starting points and for a range of values $l_{4-2}$ for each valid path starting point. To minimize the size of the mechanism, the path starting point that gives the smallest length $l_{4-2}$ was selected. The length of link $2, l_{2}$, is chosen as small as possible but long enough to result in small friction at the joints and in undisturbed tendon paths. Table III displays the chosen lengths.

\section{MECHANISM IMPLEMENTATION}

To reduce mechanism moving mass and inertia, the actuators are placed at the base. Therefore, a transmission must be employed to transmit torques and read joint angles. Among the various design options, cable drives are advantageous for their small mass and lack of backlash. Of those, friction drives, such as the ones used in [16], require a high preload, which increases joint friction and structural mass. If small loads are anticipated, then tendon drives with capstans are preferable, because they require a much smaller preload, and hence, they result in relatively small joint friction.

An important problem to be solved is how to ensure that tendon lengths do not change during a translational motion of the spherical joint. To do this, the tendon transmission path must be carefully designed. This is shown in Fig. 5. The goal is to keep the lengths of the tendons responsible for the rotational DOF constant during the translational movements of the mechanism.

Fig. 5 shows the tendon configuration with the associated pulleys for one rotational DOF. Pulleys A2 and B2 (dashed part of the tendon) are hidden behind pulleys A1 and A2.

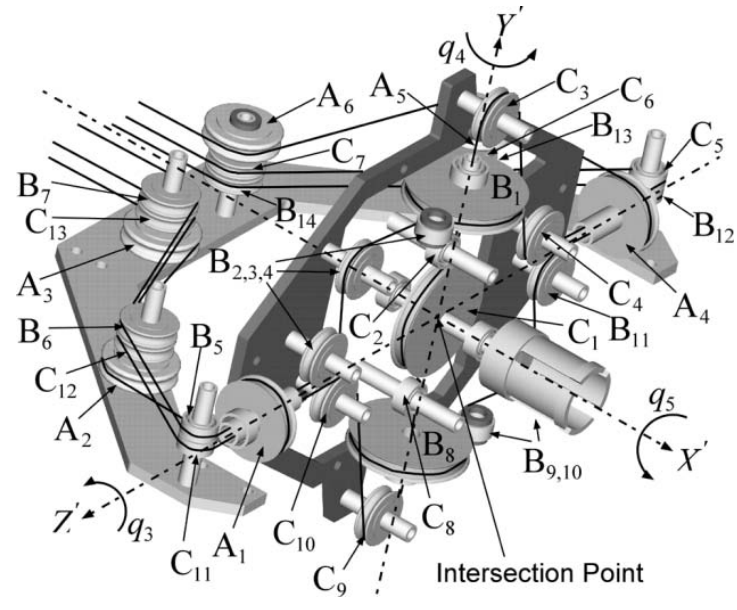

Fig. 6. Mechanism 3-DOF spherical joint with idler pulleys and tendon paths.

The basic idea employed is described next. Assume that link 1 at its starting position is vertical. In this position, the dashed part of the tendon is wrapped around pulley A2 for $270^{\circ}$ and around pulley $\mathrm{B} 2$ for $90^{\circ}$. The second part is wrapped around pulley $\mathrm{A} 1$ for $90^{\circ}$ and around pulley B1 for $270^{\circ}$. The sum is $360^{\circ}$ in each case.

Assume next that link 1 rotates by $q_{1}^{1}-q_{1}^{2}$ to a new position. Following this rotation, the dashed part of the tendon is wrapped around pulley $\mathrm{A} 2$ for $\theta_{b}=q_{1}^{1}-q_{1}^{2}$. At the same time, it is unwrapped around pulley B2 by the same angle. It is obvious that the same principle is valid for the second part of the tendon. Using this setup, we can keep the length of the tendons constant during any translational movement of link 1 .

It is also important that tendon lengths do not change during a rotational motion of the spherical joint. One way to achieve this requirement is to have the tendons travel on the rotation axis of the previous joint before they leave it.

Fig. 6 shows a schematic view of the spherical joint design. As shown in the figure, there are three groups of pulleys, the A, $\mathrm{B}$, and $\mathrm{C}$ groups, which are responsible for rotations $q_{3}, q_{4}$, and $q_{5}$, respectively. Drive pulleys $A_{1}$ and $A_{4}$ rotate around the $Z^{\prime}$ axis which is fixed in the five-bar mechanism, and therefore, the length of the tendon for joint angle $q_{3}$ is always constant.

Idler pulleys sets $B_{2}, B_{3}, B_{4}$, and $B_{9}, B_{10}$, and $B_{11}$ receive from drive pulleys $B_{1}$ and $B_{8}$, respectively, the tendons driving joint angle $q_{4}$ and guide it on the axis of rotation of the previous joint with angle $q_{3}$ and through the center of drive pulleys $A_{1}$ and $A_{4}$ to idler pulleys $B_{5}$ and $B_{12}$. It is also important to notice that the tendon from drive pulleys $B_{1}$ and $B_{8}$ to pulley sets $B_{2}$, $B_{3}, B_{4}$, and $B_{9}, B_{10}, B_{11}$ is located on the plane defined by $Y^{\prime}$ and $Z^{\prime}$ axes.

The tendon from pulley $B_{5}$ and $B_{12}$ to $B_{6}, B_{7}, B_{13}$ (not shown in Fig. 6), and $B_{14}$ respectively, is located on the plane defined by $X^{\prime}$ and $Z^{\prime}$ axes. This configuration is used in order to minimize friction between tendon and pulleys and to avoid tendon misplacement during pulley rotation.

Similarly, idler pulleys sets $C_{2}, C_{3}, C_{4}$, and $C_{8}, C_{9}, C_{10}$ guide the tendon for the third joint with angle $q_{5}$ from drive pulley $C_{1}$ through the center of drive pulleys $B_{1}$ and $B_{8}$ on the axes of the two previous joints. In such a case, motions of the two previous joints will not affect the lengths of the tendons for 


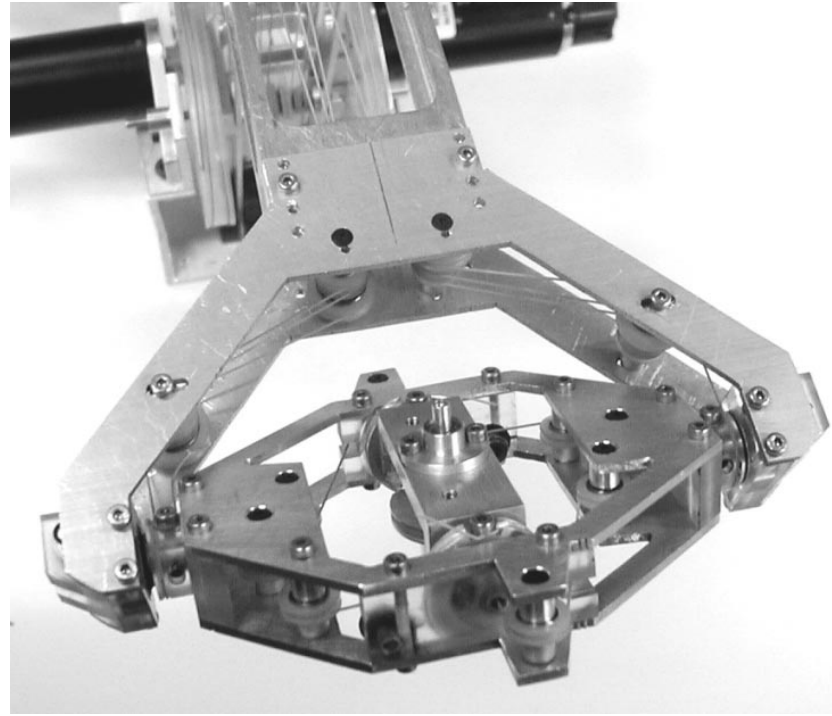

Fig. 7. Assembled spherical joint.

the last joint, and roll motions will correspond to rotations of a single motor rotor. The tendon from drive pulley $C_{1}$ to pulley sets $C_{2}, C_{3}, C_{4}$, and $C_{8}, C_{9}, C_{10}$ is located on the plane defined by $Y^{\prime}$ and $Z^{\prime}$ axes. The tendon from $C_{5}$ and $C_{11}$ to $C_{6}$ (not shown in Fig. 6), $C_{7}$, and $C_{12}, C_{13}$, respectively, is located on the plane defined by $X^{\prime}$ and $Z^{\prime}$ axes. The assembled spherical joint is shown in Fig. 7.

For the particular mechanism transmission employed, the transmission Jacobian $\mathbf{J}_{2}$ is given by

$$
\mathbf{J}_{2}=\left[\begin{array}{ccccc}
\frac{-r_{1}}{R_{1}} & 0 & 0 & 0 & 0 \\
0 & \frac{-r_{2}}{R_{2}} & 0 & 0 & 0 \\
0 & \frac{r_{2}\left(R_{3,2}-R_{3}\right)}{R_{2} R_{3}} & \frac{r_{3}}{R_{3}} & 0 & 0 \\
0 & \frac{r_{2} R_{4,2}}{R_{2} R_{4}} & 0 & \frac{r_{4}}{R_{4}} & 0 \\
0 & \frac{r_{2} R_{5,2}}{R_{2} R_{5}} & 0 & 0 & \frac{r_{5}}{R_{5}}
\end{array}\right]
$$

where $R_{i}$ is the radius of the drive pulley of the $i$ th DOF, $r_{i}$ is the radius of the $i$ th motor capstan, and $R_{i, 2}$ represents the radii of idler pulley for the $i$ th DOF located in the five bar. This Jacobian results in decoupled translations and rotations among themselves. By choosing appropriate sizes for driver pulleys and by setting $R_{3,2}=R_{3}, \mathbf{J}_{2}$ becomes close to a diagonal one.

The complete mechanism, shown in Fig. 8, was constructed mostly from aluminum. Provision has been taken so that critical parts can be replaced with ones made from composite materials and hence, further reduce its mass and friction.

The tendons are made of Dacron, a low-stretch flexible material. The tendon diameter is chosen such that the sum of the preload and dynamic tendon load is less than $10 \%$ of its breaking strength (about $200 \mathrm{~N}$ ), resulting in a diameter approximately equal to $0.5 \mathrm{~mm}$. The measured stiffness of a one-meter tendon is $3,500 \mathrm{~N} / \mathrm{m}$, with an elongation equal to $0.1 \%$. The pulleys were selected according to tendon diameter and maximum load.

The device is actuated by direct drive (no gearheads) coreless dc permanent magnet motors, powered by 10 A peak amplifiers.

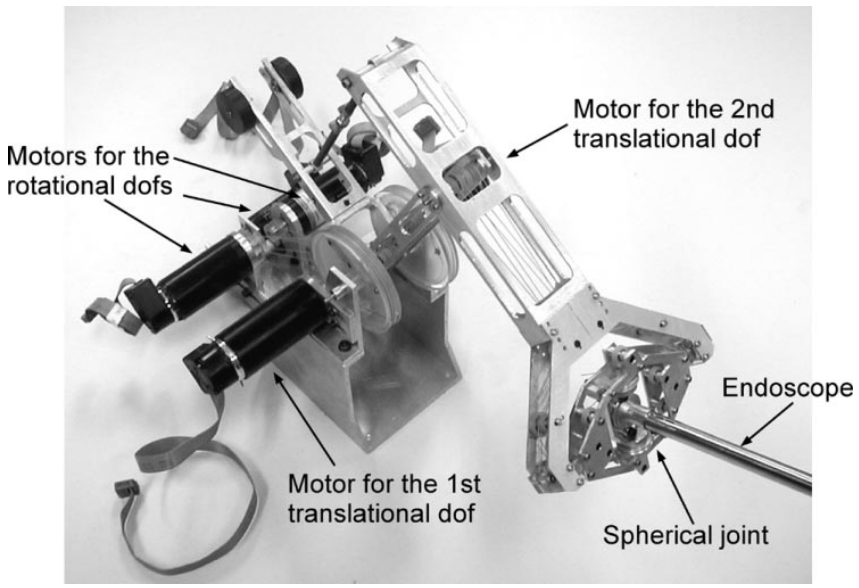

Fig. 8. Assembled 5-DOF mechanism.

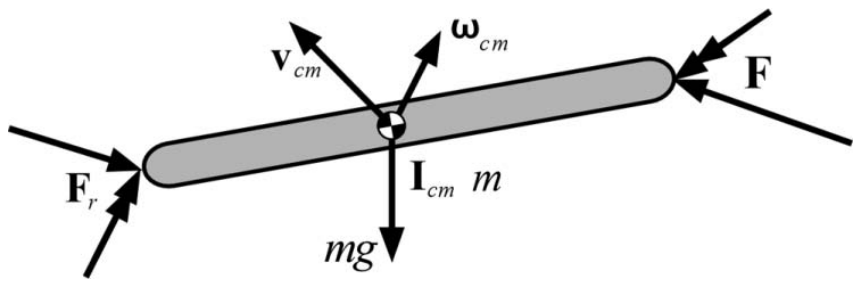

Fig. 9. Forces on the surgical tool.

The first two DOFs are powered by oversized $90 \mathrm{~W} / 48 \mathrm{~V}, 1000$ count encoder motors, the next two by $70 \mathrm{~W} / 48 \mathrm{~V}, 500$-count encoder motors, while the last DOF is powered by a $10 \mathrm{~W} / 48 \mathrm{~V}$, 500-count encoder motor.

\section{CONTROL IMPLEMENTATION}

To compute the necessary motor currents, the equations of motion of the surgical tool, see Fig. 9, are written as

$$
\mathbf{M}_{t} \dot{\mathbf{V}}_{\mathrm{cm}}+\mathbf{V}_{t}+\mathbf{G}_{t}=\mathbf{F}+\mathbf{F}_{r}
$$

where $\mathbf{F}$ is the vector of the applied forces and torques by the operator to the tool, $\mathbf{M}_{t}$ is its mass matrix, $\mathbf{V}_{t}$ contains velocity terms, $\mathbf{G}_{t}$ contains gravity terms, and $\mathbf{v}_{\mathrm{cm}}$ is the velocity of its center of mass. The vector $\mathbf{F}_{r}$ contains forces and torques which are due to tissue deformation. Since no motion is allowed in the $Z$ direction, in the virtual environment, $\mathbf{M}_{t}$ is a $5 \times 5$ matrix and the rest of the vectors have appropriate dimensions.

In general, the forces and torques $\mathbf{F}_{T}$ are functions of the position and velocity of the tool and are computed based on a simplified model of tissue deformation that has the form

$$
\mathbf{F}_{T}=\mathbf{F}_{T}(\mathbf{q} ; \mathbf{q}) \text {. }
$$

Assuming small accelerations and velocities, the motor currents applied by the power amplifiers are given by

$$
\mathbf{i}=-\left(\mathbf{K}_{T} \mathbf{K}_{\mathrm{amp}}\right)^{-1} \mathbf{J}^{T} \mathbf{F}_{T}(\mathbf{q} ; \mathbf{q})
$$

where $\mathbf{K}_{T}$, and $\mathbf{K}_{\mathrm{amp}}$ are diagonal matrices that contain motor torque constants and amplifier gains, respectively. 


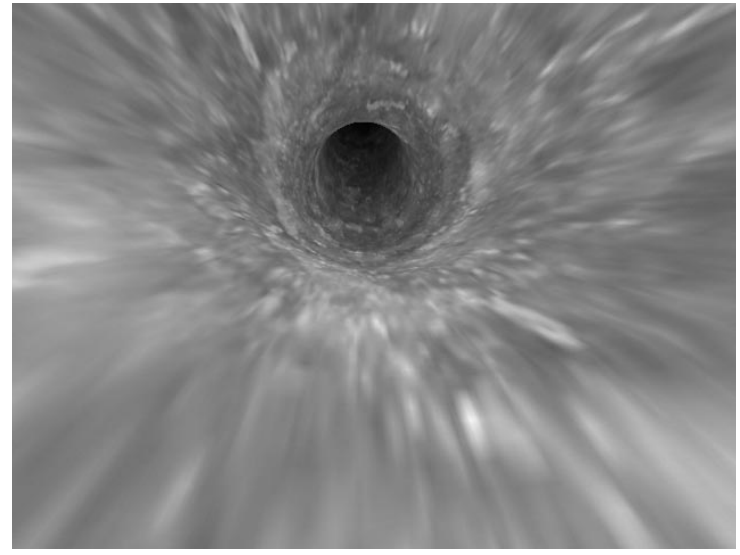

Fig. 10. Synthetic endoscope camera view of the ureter.

This haptic interface is part of a training simulator that also includes a graphics computer displaying a synchronized synthetic image of the view that would be produced by the minicamera at the tip of an endoscope. Fig. 10 shows a typical synthetic image of the ureter during endoscope insertion, as displayed on the training simulator monitor. The images are produced using spline-based nets, with appropriate textures and photorealistic techniques. The textures are constructed using photos and videos from real human tissues.

To achieve a 1-kHz force loop speed, two separate processes have been created to run on different platforms. The control loop and the calculation of the forces and torques fed to the user are implemented on a stack of PC/104 cards. These consist of a 233 $\mathrm{MHz}$ CPU module, a $24 \mathrm{MB}$ solid state flash disk, an Ethernet communications module, two encoder polling input modules, and an analog output module, that drives the motor amplifiers. This stack, along with motor amplifiers and power supplies is housed in a separate control unit. In order to have a constant refresh rate, $\mathrm{QNX}^{\circledR}$, a real-time operating system from QNX Software Systems, Ltd., is employed.

A separate process running on a separate Pentium III graphics computer equipped with the OpenGL graphics library produces the synthetic visual display of the tissues and their deformations. This process consists of two subprocesses, which are running in parallel. The first subprocess reads from the PC/104 stack the position, orientation, and velocity of the endoscope. This subprocess communicates with the one running on the PC/104 stack via Ethernet cards and the TCP/IP protocol. The second subprocess creates the series of images of endoscope minicamera views, as the endoscope moves along its virtual path.

\section{DISCUSSION}

Once the haptic system was built, experiments were run to measure its capabilities. First, its kinematic workspace was found by driving the mechanism to its limits. It was found that the spherical joint achieves rotations of $\pm 270^{\circ}$ about the $X^{\prime}$ axis, $\pm 90^{\circ}$ about the $Y^{\prime}$ axis, and $\pm 270^{\circ}$ about the $Z^{\prime}$ axis. When the endoscope is mounted to the haptic device endpoint, see Fig. 8, this workspace is reduced, but still exceeds our requirements, see Fig. 11. Fig. 12 illustrates the endoscope endpoint translational workspace with an overlay of the spherical joint workspace at selected points.

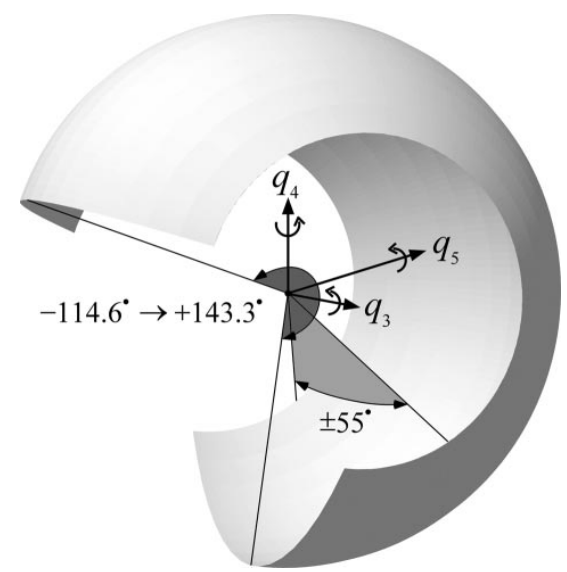

Fig. 11. Spherical joint workspace in the presence of the endoscope.

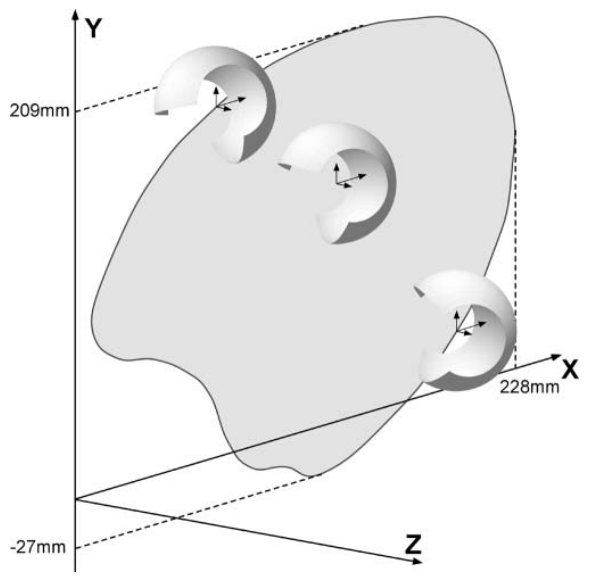

Fig. 12. Cartesian and rotational workspace limits at characteristic points.

TABLE IV

Kinematical Resolution (Average Values)

\begin{tabular}{lr}
\hline \hline Translational (X axis) & $0.046 \mathrm{~mm}$ \\
Translational (Y axis) & $0.057 \mathrm{~mm}$ \\
Rotational about the $X^{\prime}$ axis & $0.00398 \mathrm{rad}$ \\
Rotational about the $Y^{\prime}$ axis & $0.00275 \mathrm{rad}$ \\
Rotational about the 'Z' axis & $0.00279 \mathrm{rad}$ \\
\hline \hline
\end{tabular}

TABLE V

FORCE AND TORQUE RESOLUTION

\begin{tabular}{lr}
\hline \hline Force $(X, Y$ axes) & $0.00485 \mathrm{~N}$ \\
Torque about the $\mathrm{X}^{\prime}$ axis & $0.000061 \mathrm{Nm}$ \\
Torque about the $\mathrm{Y}^{\prime}$ axis & $0.000146 \mathrm{Nm}$ \\
Torque about the $Z^{\prime}$ axis & $0.000114 \mathrm{Nm}$ \\
\hline
\end{tabular}

The average kinematical resolution was found by driving the mechanism by a known distance and registering encoder output. The results are displayed in Table IV. This resolution can be improved by the use of encoders with higher than the current 500-1000 counts. Table V shows the computed mechanism force resolution.

The mass matrix of the mechanism at the main phase of the operation, see (8b), is dominated by its diagonal values

$$
\tilde{\mathbf{M}} \approx \operatorname{diag}\left(0.255,0.110,5 \times 10^{-7}, 3 \times 10^{-6}, 3 \times 10^{-5}\right)
$$



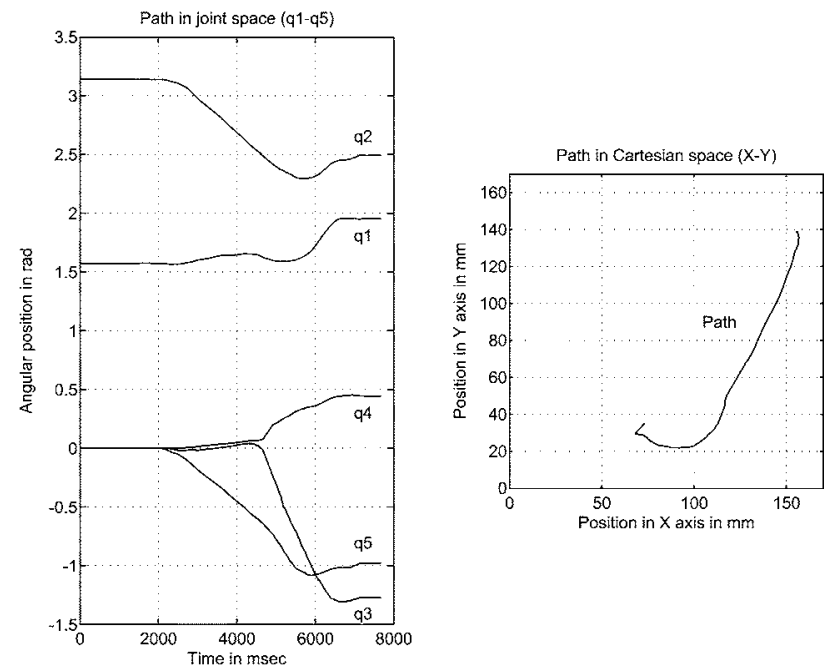

Fig. 13. Typical endoscope tip motion and actuator space histories.
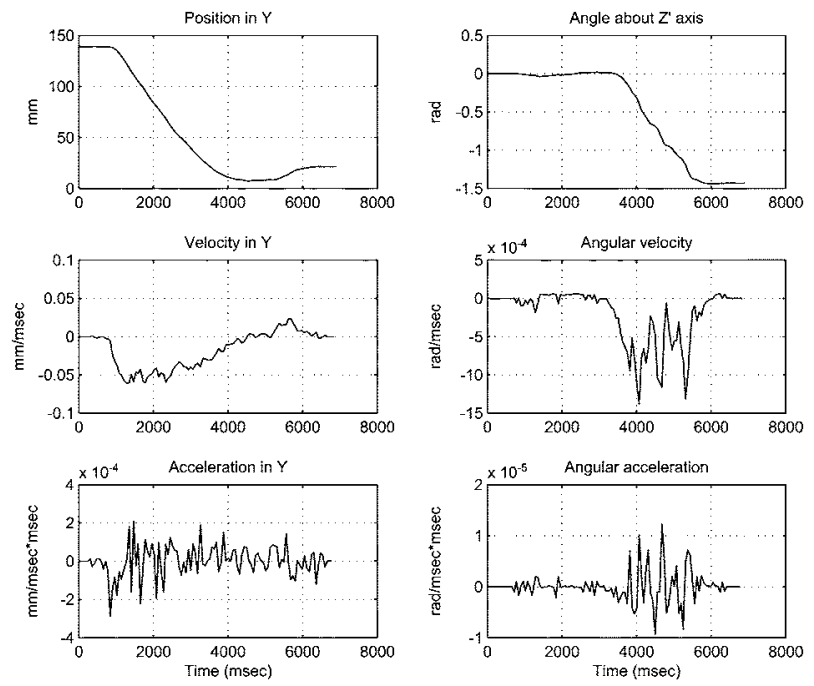

Fig. 14. $\quad Y$ and $Z^{\prime}$ axes displacements, velocities, and accelerations.

where all entries are in SI units. This result is in agreement with the experimentally determined mass and inertia, found by measuring tip forces/torques and resulting accelerations. The apparent inertias are very good as they do not exceed 0.0325 gr.m ${ }^{2}$. The apparent mass in the $X$ and $Y$ axes are about $270 \mathrm{gr}$ and $128 \mathrm{gr}$, respectively. These can be further reduced by $25 \%$, if composite materials are used for the main structural parts of the mechanism. Other improvements include the redesign of the gravity compensation and the replacement of the oversized first two DOF motors by smaller ones. However, for the operations in hand, the most critical requirement is the rotational inertia of the mechanism, which, as noted above, is minimal.

Simple strain-gauge-based experiments showed that the friction levels are about $0.09 \mathrm{~N}$ for displacements and about $4.4 \mathrm{mNm}$ for rotations. Via a mechanical stiffness experiment at the tip, the lowest resonance of the mechanism was found experimentally equal to $85 \mathrm{~Hz}$.

A typical endoscope tip motion in Cartesian space and the resulting motor angle histories are displayed in Fig. 13.
An example of a typical translational motion along the $Y$ axis and of a rotational motion about the $Z^{\prime}$ axis with the resulting velocities and accelerations is shown in Fig. 14. This figure shows that normal velocities do not exceed $0.06 \mathrm{~m} / \mathrm{s}$ and accelerations $0.3 \mathrm{~m} / \mathrm{s}^{2}$. The angular velocities are about $1.3 \mathrm{rad} / \mathrm{s}$, while angular accelerations are about $10 \mathrm{rad} / \mathrm{s}^{2}$. However, accelerations can be higher, especially during cutting, collisions, or trainee errors.

\section{CONCLUSIONS}

A new force feedback haptic mechanism was presented that is used in a training simulator for urological operations. The mechanism consists of a 2-DOF, 5-bar linkage for translations, and a 3-DOF spherical joint for rotations. To be able to reproduce very small forces and moments, the mechanism has low friction, inertia and mass, is statically balanced, and has a simple mass matrix by design. To minimize the mass of its moving parts, the actuators are located at its base and forces and moments are transmitted through the use of tendon drives with capstans and idler pulleys. Roll-pitch-yaw motions of the tool result in motions of the corresponding actuator. The mechanism design and the transmission paths were described in detail. Initial experimental results are provided and discussed. The haptic device is currently under evaluation by its potential users.

\section{ACKNOWLEDGMENT}

The authors wish to thank P. Hatzakos and L. Bahas for their assistance in CAD drawings and mechanism realization. They also wish to thank the anonymous reviewers for their constructive comments and suggestions.

\section{REFERENCES}

[1] D. Sorid and K. S. Moore, "The virtual surgeon," IEEE Spectrum, vol. 37, pp. 26-31, July 2000.

[2] E. Chen and B. Marcus, "Force feedback for surgical simulation," Proc. IEEE, vol. 86, pp. 524-530, Mar. 1998.

[3] R. C. Goertz, "Fundamentals of general-purpose remote manipulators," Nucleonics, vol. 10, pp. 36-42, 1952.

[4] J. K. Salisbury and A. M. Srinivasan, "Projects in VR. Phantom-based haptic interaction with virtual objects," IEEE Comput. Graphics Applicat. Mag., pp. 6-10, 1997.

[5] D. D'Aulignac, R. Balaniuk, and C. Laugier, "A haptic interface for a virtual exam of the human thigh," in Proc. IEEE Int. Conf. Robotics and Automation, 2000, pp. 2452-2457.

[6] T. Atsuko, H. Koichi, and K. Toyohisa, "Virtual cutting with force feedback," in Proc. Virtual Reality Annu. Int. Symp., 1998, pp. 71-75.

[7] T. H. Massie and J. K. Salisbury, "The PHANToM haptic interface: A device for probing virtual objects," in Proc. 1994 ASME Int. Mechanical Engineering Expo. Congr., Chicago, IL, 1994, pp. 295-302.

[8] E. Chen, "Six degree-of-freedom haptic system for desktop virtual prototyping applications," in Proc. 1st Int. Workshop on Virtual Reality and Prototyping, 1999, pp. 97-106.

[9] G. Burdea, G. Patounakis, V. Popescu, and R. Weiss, "Virtual realitybased training for the diagnosis of prostate cancer," IEEE Trans. Biomed. Eng., vol. 46, pp. 1253-1260, Oct. 1999.

[10] K. Kyung, D. Kwon, S. Kwon, H. S. Kang, and J. B. Ra, "Force feedback for a spine biopsy simulator with volume graphic model," in Proc. 2001 IEEE/RSJ Int. Conf. Intelligent Robots and Systems (IROS' O1), Maui, HI, Oct. 29 -Nov. 03, 2001, pp. 1732-1737.

[11] N. Pernalete et al., "Development of a robotic haptic interface to assist the performance of vocational tasks by people with disabilities," in Proc. IEEE Int. Conf. Robotics and Automation (ICRA'02), Washington, DC, May 11-15, 2002, pp. 1269-1274. 
[12] V. Hayward et al., "Freedom-7: A high fidelity seven-axis haptic device with application to surgical training," in Experimental Robotics V, A. Casals and A. T. de Almeida, Eds. New York: Springer, 1998, vol. 232, Lecture Notes in Control and Information Science, pp. 445-456.

[13] R. Baumann et al., "The pantoscope: A spherical remote-center-of-motion parallel manipulator for force reflection," in Proc. IEEE Int. Conf. Robotics and Automation, 1997, pp. 718-723.

[14] C. Baur, D. Guzzoni, and O. Georg, "VIRGY, a virtual reality and force feedback based endoscopy surgery simulator," in Proc. Medicine Meets Virtual Reality '98 (MMVR'98), 1998, pp. 110-116.

[15] U. Kühnapfel et al., "The Karlsruhe endoscopic surgery trainer as an example for virtual reality in medical education," in Minimally Invasive Therapy and Allied Technologies (MITAT). Oxford, U.K.: Blackwell, 1997, pp. 122-125.

[16] K. Salisbury et al., "The design and control of a experimental whole-arm manipulator," in The Fifth International Symposium, H. Miura and S. Arimoto, Eds. Cambridge, MA: MIT Press, 1990, pp. 233-241.

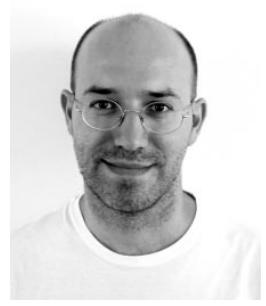

Kostas Vlachos received the B.Sc. degree in electrical engineering from the Technical University of Dresden, Dresden, Germany, in 1993. In 2000, he received the M.S. degree from the National Technical University of Athens (NTUA), Athens, Greece, where he is currently working toward the $\mathrm{Ph} . \mathrm{D}$. degree in mechanical engineering.

From 1996 to 1998 , he worked as a software analyst. His research interests are in haptic mechanisms, medical simulation, and control of robotic mechanisms.

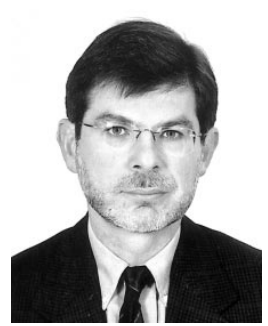

Evangelos Papadopoulos (S'83-M'91-SM'97) received the Diploma from the National Technical University of Athens (NTUA), Athens, Greece, in 1981, and the M.S. and Ph.D. degrees from the Massachusetts Institute of Technology, Cambridge, in 1983 and 1991, respectively, all in mechanical engineering.

He was an analyst with the Hellenic Navy, Athens, Greece, from 1985 to 1987. In 1991 he joined McGill University, Montreal, QC, Canada, and the Centre for Intelligent Machines (CIM) as an Assistant Professor and was tenured in 1997. Currently, he is an Associate Professor with the Mechanical Engineering Department at the NTUA. His research interests are in the area of robotics, modeling and control of dynamic systems, haptic devices, mechatronics, and design. He has published more than 85 articles in journals and conference proceedings.

Dr. Papadopoulos is a Senior Member of the AIAA, and a member of the ASME, the Technical Chamber of Greece (TEE), and the Sigma Xi.

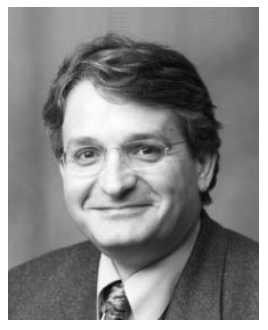

Dionissios N. Mitropoulos received the Diploma and Ph.D. degree from the Faculty of Medicine, University of Athens, Athens, Greece, in 1981 and 1983, respectively.

Currently, he is an Associate Professor with the Department of Urology, Faculty of Medicine, University of Athens. His research interests are in urological oncology, pathophysiology of prostate diseases, and minimally invasive treatment of urological diseases. He has published more than 100 articles in journals and conference proceedings.

Dr. Mitropoulos is a Board Member of the Health Care Office of the European Association of Urology. 